\title{
Electron Tomography for 3D Nanoscale Characterization of Semiconductor Materials and Devices
}

Georg Haberfehlner ${ }^{1}$, Pascale Bayle-Guillemaud ${ }^{2}$, Guillaume Audoit ${ }^{1}$, Matthew Smith ${ }^{3}$, Sam Crawford ${ }^{3}$, Silvija Gradečak ${ }^{3}$, Paul-Henry Morel $^{1}$, Thomas Ernst $^{1}$, Narciso Gambacorti ${ }^{1}$ and Pierre Bleuet ${ }^{1}$

1. CEA, LETI, MINATEC Campus, 17 rue des Martyrs, 38054 GRENOBLE Cedex 9, France.

2. CEA, INAC-UJF UMR-E Grenoble 1, SP2M, LEMMA, 17 rue des Martyrs, 38054 GRENOBLE Cedex 9, France.

3. Department of Materials Science and Engineering, Massachusetts Institute of Technology, Cambridge, Massachusetts 02139, United States.

Nanoscale devices and materials are often organized along all three dimensions of space. Though this three-dimensional (3D) arrangement can be essential for materials properties and device performance, most imaging techniques provide only two-dimensional projected representations of the investigated objects. Electron tomography can recover the full 3D information based on tilt series, which are acquired in a transmission electron microscope (TEM) [1]. This provides 3D reconstructions of samples with diameters in the range of $100 \mathrm{~nm}$ at nanometer resolution.

The most commonly used contrast technique for electron tomography on crystalline samples is Scanning TEM (STEM) using a high-angle annular dark field (HAADF) detector [1]. HAADF STEM provides contrast based on differences in the atomic number between materials. With this technique the 3D morphology of nanoscale objects can be recovered. We used this for investigating an $\mathrm{InN} / \mathrm{InGaN}$ axial heterostructure nanowire. Electron tomography allows reconstructing the shape of the nanowire and to understand the evolution the nanowire's facets during growth (see Fig. 1) [2].

The dependence of the contrast on the atomic number in HAADF STEM tomography can be used for detection of heavy elements in a lighter environment. We applied this in the investigation of selenium dopants in silicon, heavily doped by femtosecond laser irradiation [3]. Doping of silicon with chalcogens ( $\mathrm{S}, \mathrm{Se}, \mathrm{Te}$ ) to concentrations well above the solid-solubility level leads to near-unity optical absorptance in the visible and infrared range and is a promising route towards silicon-based infrared optoelectronics [4]. It has been observed that post-process annealing significantly reduces the infrared absorptance. To understand the nature of the infrared absorptance, we investigated samples before and after annealing. Precipitation of dopants with annealing was observed, linked to a reduction of the dopant concentration in solid-solution (see Fig. 2). This process is likely to be a critical mechanism for the reduction of the infrared absorptance with annealing.

In another application of electron tomography we investigated core/shell nanowires consisting of a silicon core and two shells of alumina and titanium-nitride. These nanowires form the basis of nanostructured capacitors with high capacitance density [5]. Electron tomography was applied to investigate conformity of deposition of the shells, which can influence the performance of the device. As the different materials of the nanowires have only little difference in mass, HAADF STEM did not provide sufficient contrast for reconstruction of the nanowires. Therefore we used energy-filtered TEM (EFTEM) in the low-loss range (7-27 eV), where the materials can be distinguished due to differences in their volume plasmon peaks. This allowed reconstruction of the nanowires and additionally a 3D reconstruction of local low-loss spectra (see Fig. 3) [6]. 
This work shows on several applications the interest of electron tomography for investigations of semiconductors and its contribution to answering questions about material and device properties [7].

\section{References:}

[1] P Midgley and M Weyland, Ultramicroscopy 96 (2003), p. 413.

[2] S-K Lim et al, Nano Letters, 13 (2013), p.331.

[3] G Haberfehlner et al, Microscopy and Microanalysis, accepted.

[4] M J Sher, M T Winkler and E Mazur, MRS Bulletin, 36 (2011), p. 439.

[5] P H Morel et al, Applied Physics Letters, 101 (2012), p. 083110.

[6] G Haberfehlner et al, Applied Physics Letters, 101 (2012), p. 063108.

[7] This work was supported by the MIT-France MISTI program and by the French "Recherche

Technologie de Base" (RTB) program. The authors acknowledge access to the nanocharacterization platform (PFNC) at Minatec Campus in Grenoble. We thank M.-J. Sher, M. Winkler and E. Mazur from Harvard School of of Engineering and Applied Sciences for providing fs-laser doped silicon samples.

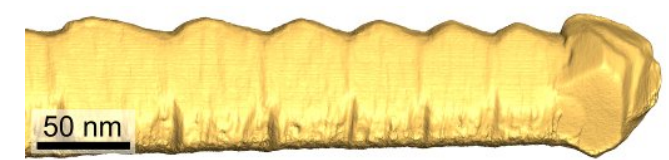

Figure 1. 3D reconstruction of an $\mathrm{InN} / \mathrm{InGaN}$ axial heterostructure nanowire [2].
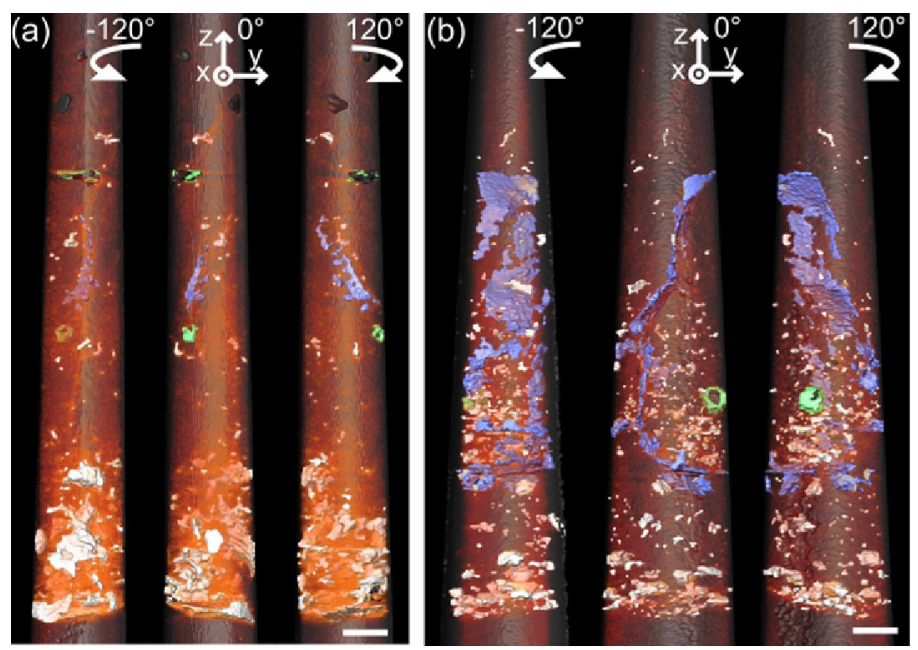

Figure 2. 3D reconstruction of Se-doped silicon (a) before annealing (b) after annealing at $950{ }^{\circ} \mathrm{C}$ for $30 \mathrm{~min}$. Segregation is observed towards precipitates (white), grain boundaries (blue) and voids (green). Different shades of red represent the dopant concentration in solid solution, scale bars are $100 \mathrm{~nm}$ [3].
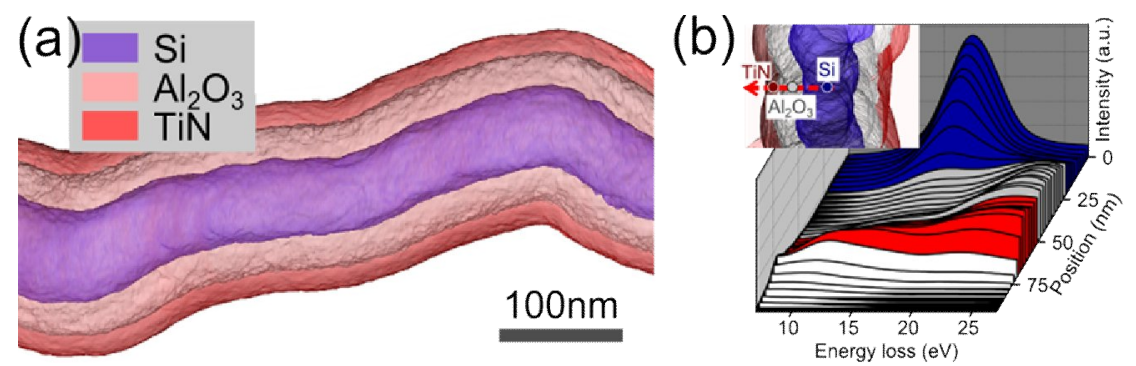

Figure 3. (a) Reconstruction of $\mathrm{Si} / \mathrm{Al}_{2} \mathrm{O}_{3} / \mathrm{TiN}$ nanowire. (b) Locally reconstructed low-loss spectra [6]. 